\title{
A System of Equations Having No Nontrivial Solutions
}

\author{
Hansraj Gupta* \\ (September 21, 1967)
}

The object of this note is to prove the THEOREM: The system of equations

$$
a_{1}^{r}+a_{2}^{r}+\ldots+a_{n-1}^{r}=b_{1}^{r}+b_{2}^{r}+\ldots+b_{n-1}^{r}, \quad r=2,3, \ldots, n
$$

has no nontrivial solutions in positive integers.

Key Words: Diophantine equations, Prouhet-Terry-Escott problem, symmetric functions.

1. The object of this note is to prove the

THEOREM: The system of equations

$$
\begin{array}{r}
a_{1}^{r}+a_{2}^{r}+\ldots+a_{n-1}^{r}=b_{1}^{r}+b_{2}^{r}+\ldots+b_{n-1}^{r}, \\
r=2,3, \ldots, n ;
\end{array}
$$

has no nontrivial solutions in positive integers.

In what follows, we write

$$
\begin{array}{ll}
A_{r} \text { for } a_{1}^{r}+a_{2}^{r}+\ldots+a_{n-1}^{r}, & \geqslant 1 ; \\
B_{r} \text { for } b_{1}^{r}+b_{2}^{r}+\ldots+b_{n-1}^{r}, & r \geqslant 1 ;
\end{array}
$$

and all small letters denote integers $\geqslant 0$ unless stated otherwise.

2. Proof of the Theorem: Let $a_{1}, a_{2}, \ldots, a_{n-1}$ be roots of the equation

$$
x^{n-1}-\lambda_{1} x^{n-2}+\lambda_{2} x^{n-3}-\ldots+(-1)^{n-1} \lambda_{n-1}=0 ;
$$

so also let $b_{1}, b_{2}, \ldots, b_{n-1}$ be roots of the equation

$$
y^{n-1}-\mu_{1} y^{n-2}+\mu_{2} y^{n-3}-\ldots+(-1)^{n-1} \mu_{n-1}=0 .
$$

Then, we have

$$
\begin{aligned}
A_{r}-\lambda_{1} A_{r-1}+\lambda_{2} A_{r-2}-\ldots & +(-1)^{r} r \lambda_{r}=0, \\
r & =1,2, \ldots, n, \ldots .
\end{aligned}
$$

with $\lambda_{r}=0 \quad$ for $r \geqslant n$.

${ }^{*}$ An invited paper. Present address: 35 Balrampur House, Allahabad-2, India.
From (4) we have

$$
r ! \lambda_{r}=\left|\begin{array}{lllllll}
A_{1} & 1 & 0 & 0 & 0 & \cdot & 0 \\
A_{2} & A_{1} & 2 & 0 & 0 & \cdot & 0 \\
A_{3} & A_{2} & A_{1} & 3 & 0 & \cdot & 0 \\
\cdot & \cdot & \cdot & \cdot & \cdot & \cdot & \cdot \\
A_{r-1} & A_{r-2} & A_{r-3} & A_{r-4} & A_{r-5} & \cdot & r-1 \\
A_{r} & A_{r-1} & A_{r-2} & A_{r-3} & A_{r-4} & \cdot & A_{1}
\end{array}\right|,
$$

with a similar expression for $r ! \mu_{r}$.

Without loss of generality, we can take

$$
A_{1}=B_{1}+h, \quad h \geqslant 0 .
$$

Writing $c_{r}$ for $r$ ! $\lambda_{r}$, and $d_{r}$ for $r ! \mu_{r}$, we then get

$$
\begin{aligned}
c_{1} & =d_{1}+h ; \\
c_{2} & =A_{1}^{2}-A_{2}=B_{1}^{2}+2 h B_{1}+h^{2}-B_{2}=d_{2}+2 h d_{1}+h^{2}, \\
c_{3} & =A_{1}^{3}-3 A_{1} A_{2}+2 A_{3}, \\
& =\left(B_{1}+h\right)^{3}-3\left(B_{1}+h\right) B_{2}+2 B_{3}, \\
& =d_{3}+3 h d_{2}+3 h^{2} d_{1}+h^{3} .
\end{aligned}
$$

Assuming that

$$
c_{k}=d_{k}+\left(\begin{array}{l}
k \\
1
\end{array}\right) h d_{k-1}+\left(\begin{array}{l}
k \\
2
\end{array}\right) h^{2} d_{k-2}+\ldots+\left(\begin{array}{l}
k \\
k
\end{array}\right) h^{k},
$$

for $k=1,2,3, \ldots, r-1,(4)$ gives 
$B_{r}-\frac{1}{1 !}\left(d_{1}+h\right) B_{r-1}+\frac{1}{2 !}\left(d_{2}+2 h d_{1}+h^{2}\right) B_{r-2}-\ldots$

$$
\begin{aligned}
+\frac{(-1)^{r-1}}{(r-1) !}\left\{d_{r-1}+\left(\begin{array}{c}
r-1 \\
1
\end{array}\right) h d_{r-2}\right. \\
\left.+\ldots+\left(\begin{array}{c}
r-1 \\
r-1
\end{array}\right) h^{r-1}\right\}\left(B_{1}+h\right) \\
+\frac{(-1)^{r}}{r !} r c_{r}=0, \quad n \geqslant 2 .
\end{aligned}
$$

Since

$$
\begin{aligned}
B_{k}-\frac{d_{1}}{1 !} B_{k-1}+\frac{d_{2}}{2 !} B_{k-2}-\ldots & +\frac{(-1)^{k-1}}{(k-1) !} d_{k-1} B_{1} \\
+ & \frac{(-1)^{k}}{k !} k d_{k} \equiv 0, \quad k>1 ;
\end{aligned}
$$

we get

$$
c_{r}=d_{r}+\left(\begin{array}{l}
r \\
1
\end{array}\right) h d_{r-1}+\ldots+\left(\begin{array}{l}
r \\
r
\end{array}\right) h^{r} .
$$

Symbolically, this result can be written in the form

$$
c_{r} \simeq(d+h)_{r}, \quad r=1,2, \ldots, n-1, n, \ldots .
$$

In particular

$$
c_{n}=d_{n}+\left(\begin{array}{c}
n \\
1
\end{array}\right) h d_{n-1}+\ldots+\left(\begin{array}{l}
n \\
n
\end{array}\right) h^{n} .
$$

Since $c_{n}=0=d_{n}$, we must have

$$
h\left\{\left(\begin{array}{l}
n \\
1
\end{array}\right) d_{n-1}+\ldots+\left(\begin{array}{l}
n \\
n
\end{array}\right) h^{n-1}\right\}=0 .
$$

' H. Schmidt. Über einige diophantische Aufgaben 3 und 4 Grades. Jber. Deutsch Math. Verein 67 (1964/65) Abt. 1, 2-13.
As the expression in the curly brackets is positive, (8) can hold only with $h=0$. This shows that $c_{i}=d_{i}$, i.e., $\lambda_{i}=\mu_{i}$ for $i=1,2,3, \ldots, n-1$. Hence eqs (2) and (3) have the same roots and $a$ 's are $b$ 's in some order.

Alternatively

$$
\begin{aligned}
A_{n} & =\lambda_{1} A_{n-1}-\lambda_{2} A_{n-2}+\ldots+(-1)^{n-2} \lambda_{n-1} A_{1}, \\
& =\frac{c_{1}}{1 !} A_{n-1}-\frac{c_{2}}{2 !} A_{n-2}+\ldots+(-1)^{n-2} \frac{c_{n-1}}{(n-1) !} A_{1}, \\
& =\frac{1}{1 !}\left(d_{1}+h\right) B_{n-1}-\frac{1}{2 !}\left(d_{2}+2 h d_{1}+h^{2}\right) B_{n-2}+\ldots
\end{aligned}
$$

$$
+\frac{(-1)^{n-2}}{(n-1) !}\left\{d_{n-1}+\left(\begin{array}{c}
n-1 \\
1
\end{array}\right) h d_{n-2}+\ldots\right.
$$$$
\left.+\left(\begin{array}{l}
n-1 \\
n-1
\end{array}\right) h^{n-1}\right\}\left(B_{1}+h\right)
$$

$$
\begin{aligned}
& =B_{n}+\frac{h}{(n-1) !}\left\{\left(\begin{array}{l}
n \\
1
\end{array}\right) d_{n-1}+\left(\begin{array}{l}
n \\
2
\end{array}\right) h d_{n-2}+\ldots\right. \\
& \left.+\left(\begin{array}{l}
n \\
n
\end{array}\right) h^{n-1}\right\} .
\end{aligned}
$$

Since $A_{n}=B_{n}$, the result follows as before.

3. H. Schmidt ${ }^{1}$ has proved a special case of our theorem when $n=3$.

Proceeding on the same lines as we have done in section 2 , it can be proved that any $(n-1)$ of the $n$ equations

$$
A_{r}=B_{r}, 1 \leqslant r \leqslant n,
$$

have only nontrivial solutions. 